\title{
Anisotropic Hexagonal Particles Induced by the Double-Solvent Swelling Method
}

Liujun Song, Xiaofeng Huang, Xiaoafei Chen, Li Zhong, Xiang Jiang*, Xinya Zhang*

School of Chemistry and Chemical Engineering, Guangdong Provincial Key Lab of Green Chemical

Product Technology, South China University of Technology, Guangzhou 510640, P.R. China

*Xiang Jiang, E-mail: jiangx@scut.edu.cn

*Xinya Zhang, E-mail: cexyzh@scut.edu.cn 


\begin{tabular}{l|ll}
\hline Element & Weight $\%$ & Atomic\% \\
& & \\
C K & 63.37 & 76.25 \\
O K & 12.61 & 11.39 \\
Si K & 24.03 & 12.36 \\
& & \\
Totals & 100.00 & \\
\hline
\end{tabular}

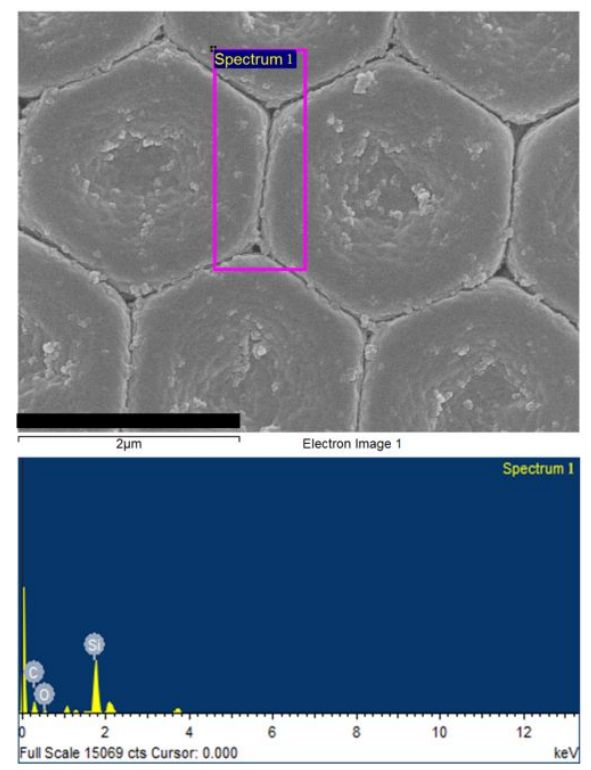

Figure S1. The energy-dispersive spectrometry (EDS) of the swollen particle arrays, showing the hexagonal particles were coated by silica. The swollen arrays are corresponding to Figure $3 \mathrm{c}$ in the manuscript
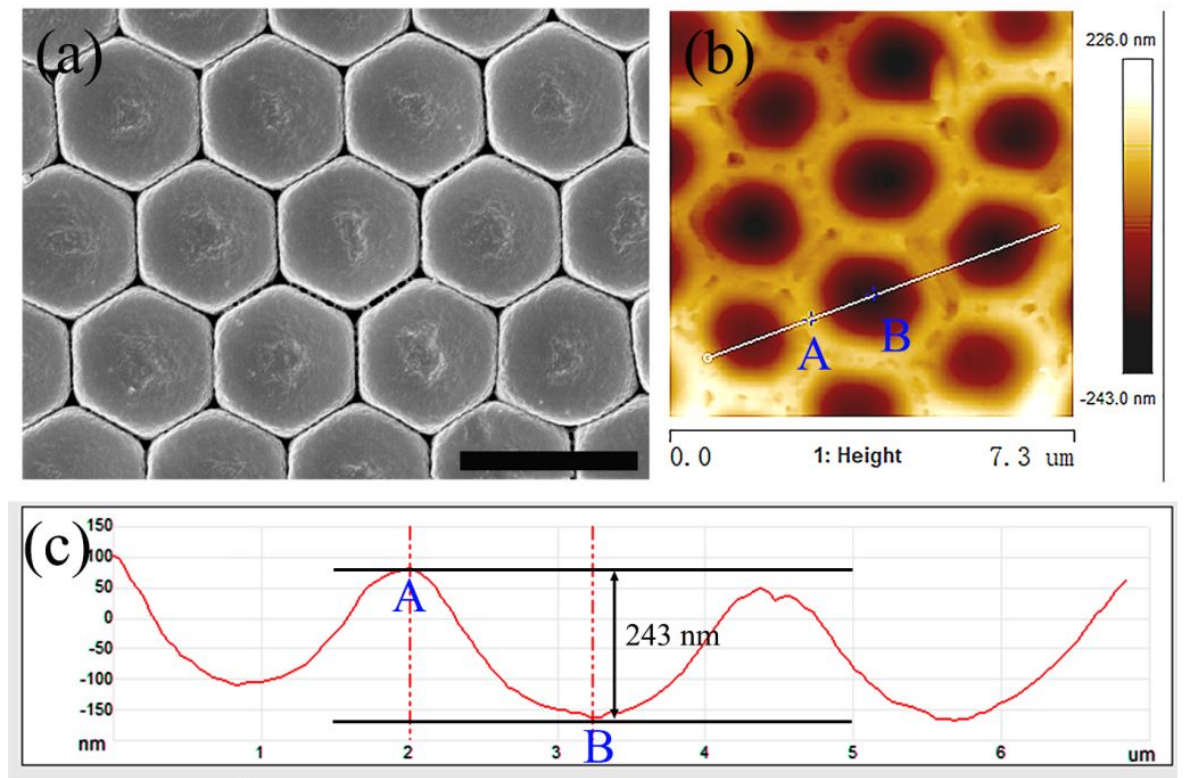

Figure S2. SEM image and their corresponding AFM image of the particle arrays treated under the double-solvent system for $90 \mathrm{~s}, 60{ }^{\circ} \mathrm{C}$. The scale bar is $3 \mu \mathrm{m}$. 

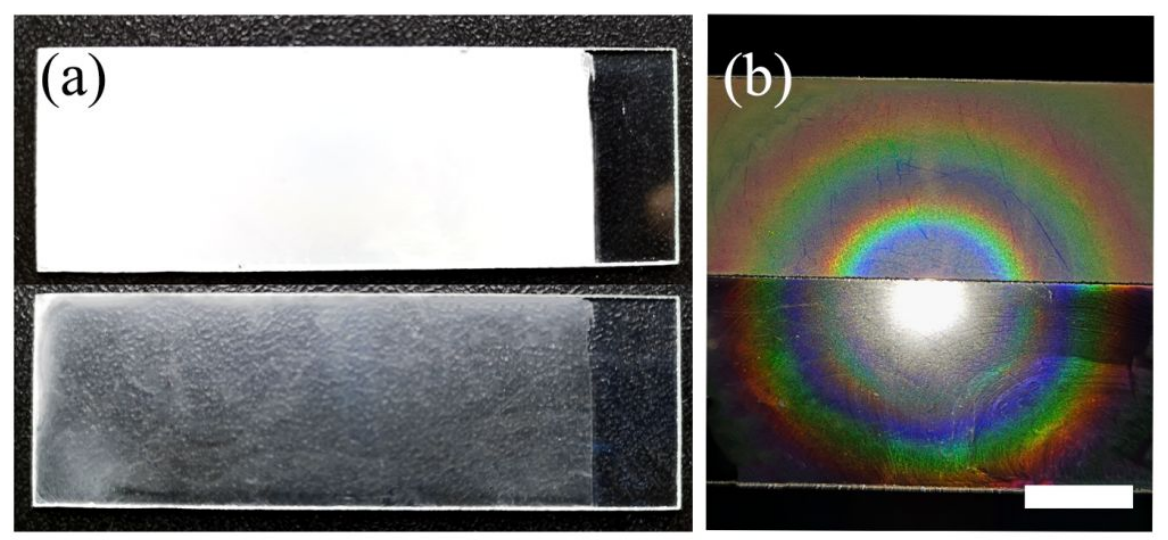

Figure S3. (a) The appearance of the particle array before (white one) and after (transparent one) swollen by the double-solvent system. (b) the bright and beautiful optical diffraction of (a) under a conical light, showing the high-quality of the particle arrays. The scale bar is $1 \mathrm{~cm}$.
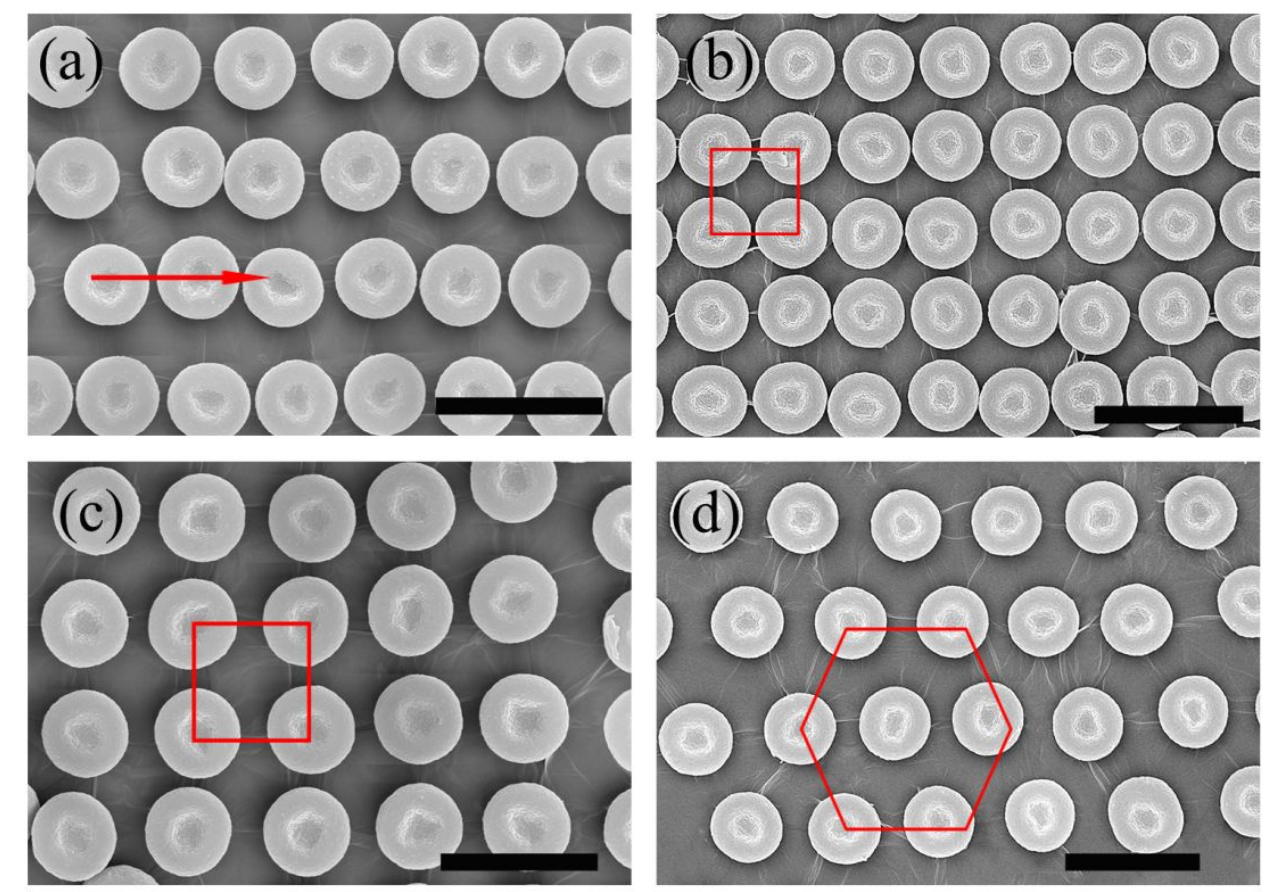

Figure S4. The non-close-packed colloidal arrays with a different lattice structure before swollen.

(a) Quasi-one-dimension chain-like arrays. (b and c) Square arrays with a different lattice constant.

(d) Hexagonal non-close-packed colloidal arrays. 